\title{
Estimación de heterosis individual en cuyes (Cavia porcellus) F1 cruces de los genotipos Peru, Inti y Andina
}

\author{
ESTIMATION OF INDIVIDUAL HETEROSIS IN GUINEA PIGS (Cavia porcellus) F1 CROSSES \\ of Peru, Inti And Andina genotypes \\ Elmer Meza R..$^{1,4}$, Yiyson Rojas Y. ${ }^{2}$, Jorge Raymondi C. ${ }^{3}$, Cesar Olaguivel F. ${ }^{1}$
}

\section{Resumen}

El presente estudio se realizó en la E.E.A. Canaán del INIA(Ayacucho, Perú) con el objetivo de estimar la heterosis individual en cuyes F1 procedentes de los cruces entre los genotipos Perú (P), Andina (A) e Inti (I) para las características de peso al beneficio, velocidad de crecimiento, rendimiento de carcasa, grasa de deposición y conversión alimenticia. Se obtuvo información de 115 descendientes entre puros y cruzados de los genotipos PxA, AxP y PxI, siendo criados como grupo contemporáneo durante toda la fase de recría. La incidencia del tamaño de camada, sexo de la cría y número de parto de la madre sobre las variables en estudio fue analizada a través de un modelo de efectos fijos, estimándose factores de corrección por mínimos cuadrados para aquellos que resultaron tener incidencia significativa $(p<0.05)$. La heterosis individual fue estimada como el desvío de los promedios de los cruzamientos recíprocos frente al promedio de los parentales puros. La heterosis para el carácter peso al beneficio, estimada a nivel de los cruces PxI (genotipo Perú como vía paterna) y el recíproco PxA, fue de $11.90 \mathrm{~g}(1.5 \%)$ y $10.25 \mathrm{~g}(1.3 \%)$, respectivamente; en tanto que la velocidad de crecimiento tuvo una heterosis de $0.39 \mathrm{~g} /$ día (3.9\%) para el cruce PxI (genotipo Perú como vía paterna), no observándose niveles apreciables de heterosis para las otros caracteres evaluados. Se concluye que el cruzamiento entre cuyes mejorados no produce niveles significativos de heterosis individual a nivel de los caracteres relacionados con la producción de carne.

Palabras clave: cruzamiento; heterosis; cuy; genotipo; efecto fijo

\footnotetext{
${ }^{1}$ Escuela Profesional de Medicina Veterinaria, Facultad de Ciencias Agrarias, Universidad Nacional San Cristóbal de Huamanga, Ayacucho, Perú

${ }^{2}$ Escuela Profesional de Agronomía, Facultad de Ciencias Agrarias, Universidad Nacional San Cristóbal de Huamanga, Ayacucho, Perú

${ }^{3}$ Estación Experimental Agraria Canaán, Instituto Nacional de Innovación Agraria, Ayacucho, Perú

${ }^{4}$ E-mail: elzar2112@hotmail.com
}

Recibido: 5 de septiembre de 2017

Aceptado para publicación: 8 de marzo de 2018 
The present study was carried out in the E.E.A. Canaan from INIA (Ayacucho, Peru) with the objective of estimating the individual heterosis in F1 guinea pigs from the crosses between the genotypes Peru (P), Andean (A) and Inti (I) for the characteristics of weight at slaughtering, growth, carcass yield, fat deposition and feed conversion. Information was obtained from 115 descendants between pure lines and crosses of the genotypes PxA, AxP and PxI, being reared as contemporary groups throughout the rearing phase. The incidence of the fixed effects of litter size, sex of the offspring and number of parturition of the mother on the variables under study was analysed through a model of fixed effects, estimating correction factors by least squares for those that had significant incidence $(\mathrm{p}<0.05)$. The individual heterosis was estimated as the deviation of the mean of the reciprocal cross compared to the average of the pure parents. The heterosis for the weight at slaughtering character, estimated at the level of crosses PxI (genotype Peru as a paternal route) and the reciprocal PxA, was $11.90 \mathrm{~g}(1.5 \%)$ and 10.25 $\mathrm{g}(1.3 \%)$, respectively; while the growth rate had a heterosis of $0.39 \mathrm{~g} /$ day $(3.9 \%)$ for the PxI cross (Peru genotype as a paternal route). No appreciable levels of heterosis were observed for the other traits. It is concluded that the crossing between improved guinea pigs does not produce significant levels of individual heterosis at the level of the characters related to meat production.

Key words: crossbreeding; heterosis; guinea pig; genotype; fixed effect

\section{INTRODUCCIÓN}

Las iniciativas de mejora genética de los rasgos cuantitativos de los grupos genéticos de cuyes (Cavia porcellus) existentes en el Perú en las últimas décadas se han enfocado mayormente a la mejora del peso vivo, la ganancia de peso, la precocidad y el tamaño de camada por medio de la selección (Chauca, 2007). Sin embargo, se requiere prestar atención a otros rasgos de crucial importancia y que suelen contribuir en la mejora de la eficiencia de la producción de carne de la mayoría de las especies de importancia zootécnicas, tales como la conversión alimenticia, conformación de masa muscular, nivel de marmoleo y rendimiento de carcasa (Bianchi et al., 2001), pero que, por lo general, no se toman en cuenta debido a la dificultad de su medición (Rivas y Rico, 2014).

La mayoría de los rasgos cuantitativos asociados con la producción de carne suelen tener una heredabilidad de media a alta y, por tanto, responden muy bien a la selección. Esto permite a su vez, aprovechar los efectos aditivos de los genes relacionados con su expresión (Magofke y García, 2002); sin embargo, algunos atributos, sobre todo los de naturaleza reproductiva, suelen tener baja heredabilidad, por lo que requieren ser mejorados por medio del cruzamiento, salvo que sea la complementariedad el objetivo deseado (Gardner et al., 1999; Magofke y García, 2001).

El cruzamiento, a diferencia de la selección, permite aprovechar los efectos derivados de las interacciones génicas, lo que podría dar lugar a la manifestación de heterosis o vigor híbrido, siempre y cuando se dispongan de razas o poblaciones genéticamente diferenciados (Cardellino y Rovira, 1987; Magofke y García, 2002). Para la estimación de la heterosis se requerirá evaluar en la descendencia híbrida los caracteres de interés y compararlos con los de la descendencia contemporánea de los parentales puros apareados en forma dialélicos (Falconer y Mackay, 2001). Posterior a la corrección de los datos 
por los factores ambientales sistemáticos, se procede a determinar la diferencia de las medias fenotípicas de los grupos genéticos involucrados, cuyos desvíos son atribuidos al efecto de la expresión de la heterosis (Gardner et al, 1999; Magofke y García, 2002).

A diferencia de otras especies de interés pecuario, son escasos los reportes científicos de estimación de heterosis realizados en cuyes (Muscari, 1993). Por otro lado, las pocas experiencias de cruzamientos han estado mayormente orientados a evaluar la media fenotípica de los híbridos sobre la base de líneas, ecotipos nativos y/o grupos genéticos mejorados, y solamente para caracteres de peso vivo individual, tamaño y peso de camada al nacimiento y al destete (Chauca, 2007). Sin embargo, dichas experiencias han sido generalmente reportadas a nivel de resúmenes de congresos científicos, siendo limitado el registro en revistas científicas especializadas (Chauca et al., 2008).

El objetivo del presente estudio fue estimar la heterosis individual para características productivas relacionadas con la producción de carne en cuyes cruzados F1, a partir de los genotipos mejorados Perú, Andina e Inti.

\section{Materiales y Métodos}

El presente estudio se realizó en la unidad de animales menores de la Estación Experimental Agraria (E.E.A) Canaán del Instituto Nacional de Innovación Agraria (INIA), Huamanga, Ayacucho, Perú. Se evaluaron 115 descendientes entre puros y cruzados de 14 días de edad, nacidos entre finales de enero a inicios de marzo de 2011. La cantidad de descendientes según genotipo evaluado se muestra en el Cuadro 1.

Toda la descendencia fue criada como grupo contemporáneo en un ambiente acondicionado con 12 pozas de $1.0 \times 1.5 \mathrm{~m}$, some-
Cuadro 1. Número de cuyes evaluados según genotipo

\begin{tabular}{llcc}
\hline Genotipo del & Genotipo de & \multicolumn{2}{c}{ Descendencia } \\
\cline { 3 - 4 } padre & la madre & Genotipo & $\mathrm{n}$ \\
\hline Perú (P) & Perú (P) & (PxP) & 20 \\
Perú (P) & Andina (A) & (PxA) & 18 \\
Andina (A) & Perú (P) & $($ AxP) & 19 \\
Andina (A) & Andina (A) & (AxA) & 19 \\
Inti (I) & Inti (I) & (IxI) & 19 \\
Perú (I) & Inti (I) & (PxI) & 20 \\
\hline Total & & & 115 \\
\hline
\end{tabular}

tidos a un mismo protocolo de manejo y alimentación durante las 12 semanas que duró la fase de recría. La diferencia de edad entre el primer y último nacimiento fue de 38 días. Las variables evaluadas fueron: peso al beneficio (PB), velocidad de crecimiento (VC), rendimiento de carcasa (RC), grasa de deposición $(\mathrm{GD})$ y conversión alimenticia (CA).

El PB (en gramos) de cada animal fue medido a los 85 días de edad, la VC (en g/día) fue determinada como la diferencia entre el último registro de peso vivo y el peso al destete dividido entre los días de crecimiento. La CA se obtuvo dividiendo el consumo de alimento acumulado del grupo (en términos de materia seca) entre el incremento de peso medio del grupo. El RC (en porcentaje) fue determinado como el cociente entre el peso de la carcasa limpia eviscerada y el peso al beneficio. Para la determinación de la GD (en porcentaje) se pesaron los cúmulos de grasa subcutánea (en gramos) extraídos manualmente de la zona de la cruz del animal en el momento del beneficio y se dividió entre el peso de la carcasa (en gramos). Para todos efectos de pesado se utilizó una balanza digital de $\pm 5 \mathrm{~g}$ de precisión.

El análisis de la información consistió en establecer la incidencia de los efectos fijos identificados (sexo de la cría, tamaño de- 
Cuadro 2. Promedios fenotípicos corregidos para el carácter peso al beneficio (g) según grupo genético evaluado

\begin{tabular}{lccc}
\hline Genotipo no & \multicolumn{3}{c}{ Genotipo no híbrido (madres) } \\
\cline { 2 - 4 } híbrido (padres) & Perú (P x P) & Andina (A x A) & Inti (I X I) \\
\hline Perú (P x P) & P x P & P x A & P x I \\
& $(790.7)$ & $(855.8)$ & $(819.6)$ \\
Andina (A x A) & $\mathrm{A} \times \mathrm{P}$ & $\mathrm{A} \times \mathrm{A}$ & $\mathrm{A} \times \mathrm{I}$ \\
& $(750.6)$ & $(795.1)$ & $(\mathrm{n} . \mathrm{d})$ \\
Inti (I x I) & $\mathrm{I} \times \mathrm{P}$ & $\mathrm{I} \times \mathrm{A}$ & $\mathrm{I} \times \mathrm{I}$ \\
& $(\mathrm{n} . \mathrm{d})$ & $(\mathrm{n} . \mathrm{d})$ & $(824.7)$ \\
\hline
\end{tabular}

n.d.= No se determinó

Cuadro 3. Heterosis individual promedio estimado para el carácter peso al beneficio, a nivel de los cruzamientos efectuados

\begin{tabular}{|c|c|c|c|c|}
\hline \multirow{2}{*}{ Genotipos } & \multirow{2}{*}{$\begin{array}{l}\text { Promedio de la } \\
\text { descendencia no } \\
\text { híbrida }\end{array}$} & \multirow{2}{*}{$\begin{array}{l}\text { Promedio de la } \\
\text { descendencia } \\
\text { híbrida }\end{array}$} & \multicolumn{2}{|c|}{ Heterosis } \\
\hline & & & Gramos & $\%$ \\
\hline Perú - Andina & 792.9 & $803.2^{1}$ & 10.25 & 1.3 \\
\hline Perú - Inti & 807.7 & $819.6^{2}$ & 11.90 & 1.5 \\
\hline Andina - Inti & 809.9 & n.d. & n.d. & n.d. \\
\hline
\end{tabular}

n.d.= No se determinó

${ }^{1}$ Promedio fenotípico de los cruzamientos recíprocos (PxA y AxP)

${ }^{2}$ Promedio fenotípico del cruce que tuvo al genotipo Perú como vía paterna

Cuadro 4. Promedios fenotípicos corregidos para el carácter velocidad de crecimiento (g) según grupos genético evaluado

\begin{tabular}{lccc}
\hline Genotipo no & \multicolumn{3}{c}{ Genotipo no hibrido (madres) } \\
\cline { 2 - 4 } híbrido (padres) & Perú (P x P) & Andina (A x A) & Inti (I x I) \\
\hline Perú (P x P) & $\mathrm{P} \times \mathrm{P}$ & $\mathrm{P} \times \mathrm{A}$ & $\mathrm{P} \times \mathrm{I}$ \\
& $(10.2)$ & $(10.0)$ & $(10.3)$ \\
Andina (A x A) & $\mathrm{A} \times \mathrm{P}$ & $\mathrm{A} \times \mathrm{A}$ & $\mathrm{A} \times \mathrm{I}$ \\
& $(9.2)$ & $(9.6)$ & (n.d) \\
Inti (I x I) & $\mathrm{I} \times \mathrm{P}$ & $\mathrm{I} \times \mathrm{A}$ & $\mathrm{I} \times \mathrm{I}$ \\
& (n.d.) & (n.d.) & $(9.7)$ \\
\hline
\end{tabular}

n.d.= No se determinó 
camada al nacimiento y número de parto de la madre) sobre las variables en estudio, con base al siguiente modelo aditivo lineal: $Y_{i j k l}=$ $\mu+S_{i}+T_{j}+N_{k}+b_{l}\left(x_{1}-x\right)+e_{i j k l}$, donde $Y_{i j k l}$ $=$ Variable observada en la 1-ésimo cría de iésimo sexo, j-ésimo tipo de parto y k-ésimo numero de parto de la madre; $S_{i}=$ Efecto del i-ésimo sexo de la cría; $T_{j}=$ Efecto del $\mathrm{j}$ ésimo tipo de parto de la cría; $N_{k}=$ Efecto del $k$-ésimo número de parto de la madre; $b_{1}=$ Regresión del peso al destete sobre la variable respuesta evaluada; $x_{1}=$ Efecto de la variable independiente peso al nacimiento; $x$ $=$ Promedio de la variable independiente peso al destete; $e_{i j k l}=$ Efecto aleatorio del error asociado a cada observación.

La información fue procesada y analizada mediante el uso del software SAS v. 9.2. No se consideró el efecto de la estación del año ya que toda la descendencia F1 cruzada y no híbrida fue manejada como grupo contemporáneo.

Sobre la base de aquellos efectos fijos que tuvieron incidencia significativa $(\mathrm{p}<0.05)$, se elaboraron submodelos que permitieron estimar mediante el método de mínimos cuadrados los respectivos factores de corrección (Gutiérrez, 2010). El predictor lineal usado fue el siguiente: $b=\left[Y^{\prime} X\right]^{-}\left[Y^{\prime} Y\right]$, donde $b$ $=$ Vector solución de incidencia de efectos fijos desconocidos (factores de corrección); $Y=$ Vector de variables observadas; $Y^{\prime}=$ Transpuesta del vector de variables observadas; $X=$ Matriz de incidencia de efectos fijos conocidos de efectos significativos.

La resolución del sistema de ecuaciones lineales se efectuó con el uso del software Matlab v. 7.5 (Matlab, 2007). Con los factores de corrección hallados, se procedió a ajustar los datos de cada animal según el nivel del factor al que estuvo asociado, obteniéndose posteriormente las medias fenotípicas corregidas para los diferentes cruces y variables estudiadas. Finalmente, la heterosis individual se obtuvo mediante la diferencia entre el promedio de los cruzamien- tos recíprocos respecto al promedio de los descendientes puros (Magofke y García, 2002).

\section{Resultados y Discusión}

\section{Peso al Beneficio}

En los cuadros 2 y 3 se presentan los promedios fenotípicos corregidos para el carácter peso al beneficio a nivel de los grupos genéticos evaluados, así como los respectivos desvíos fenotípicos de los genotipos cruzados respecto al promedio de los parentales no híbridos. Se puede observar que la descendencia PxA fue el que tuvo el mejor promedio fenotipo $(855.8 \mathrm{~g})$ con relación a su recíproco AxP (750.6 g) y al cruce PxI (819.6 $\mathrm{g})$, lo que implica un desvío fenotípico promedio o heterosis individual de $62.8 \mathrm{~g}(7.9 \%)$, -42.4 y 11.9 g (1.5\%), respectivamente. Por otro lado, la heterosis individual promedio obtenido en los cruzamientos recíprocos PxA y AxP fue de $10.25 \mathrm{~g}(1.3 \%)$, no determinándose para aquellos cruces que tuvieron al genotipo Inti como parental, con excepción del cruce PxI, posiblemente debido al limitado número de animales disponibles para el estudio.

La heterosis individual promedio encontrado para el carácter PB a nivel de los tres grupos de descendientes híbridos $\mathrm{F} 1$ resultó ser ínfima, con excepción del cruce que tuvo como vía paterna al genotipo Perú y como vía maternal al genotipo Andina, lo que indicaría una importante contribución del efecto maternal de este genotipo sobre la expresión del carácter (Dulanto, 1999; Angulo, 2013). Aun así, los desvíos fenotípicos hallados resultan ser de limitado impacto como para contribuir en la mejora productiva y económica del sistema de producción de cuyes; a razón de que durante la comercialización del producto no suele compensarse con un mayor ingreso marginal el diferencial de peso vivo o de carcasa favorable en aquellos animales con evidencia de una mayor superioridad genotípica. 
Cuadro 5. Heterosis individual promedio estimado para el carácter velocidad de crecimiento (gr/día), a nivel de los cruzamientos efectuados

\begin{tabular}{lcccc}
\hline \multirow{2}{*}{ Genotipos } & $\begin{array}{c}\text { Promedio de la } \\
\text { descendencia no } \\
\text { híbrida }\end{array}$ & \begin{tabular}{c} 
Promedio de la \\
descendencia \\
\cline { 4 - 5 }
\end{tabular} & 9.89 & \multicolumn{2}{c}{ Heterosida } \\
\hline Perú - Andina & 9.95 & Gramos & $\%$ \\
Perú - Inti & 9.66 & $10.34^{1}$ & -0.29 & -3.0 \\
Andina - Inti & 9.66 & n.d. & 0.39 & 3.9 \\
\hline
\end{tabular}

n.d.= No se determinó

${ }^{1}$ Promedio fenotípico de los cruzamientos recíprocos (PxA y AxP)

${ }^{2}$ Promedio fenotípico del cruce que tuvo al genotipo Perú como vía paterna

Cuadro 6. Promedios fenotípicos corregidos para el carácter rendimiento de carcasa (\%) según el grupo genético

\begin{tabular}{lccc}
\hline $\begin{array}{l}\text { Genotipo no } \\
\text { hibrido (padres) }\end{array}$ & \multicolumn{3}{c}{ Genotipo no híbrido (madres) } \\
\cline { 2 - 4 } & Perú (P x P) & Andina (A x A) & Inti (I x I) \\
\hline Perú (P x P) & P x P & P x A & P x I \\
& $(66.3)$ & $(65.4)$ & $(65.3)$ \\
Andina (A x A) & A x P & A x A & A x I \\
& $(65.2)$ & $(66.8)$ & (n.d) \\
Inti (I x I) & I x P & I x A & I x I \\
& (n.d) & (n.d) & $(66.9)$ \\
\hline
\end{tabular}

n.d.= No se determinó

Cuadro 7. Heterosis individual promedio estimado para el carácter rendimiento de carcasa $(\%)$

\begin{tabular}{|c|c|c|c|c|}
\hline \multirow{2}{*}{ Genotipos } & \multirow{2}{*}{$\begin{array}{c}\text { Promedio de la } \\
\text { descendencia no } \\
\text { híbrida }\end{array}$} & \multirow{2}{*}{$\begin{array}{c}\text { Promedio de la } \\
\text { descendencia } \\
\text { híbrida }\end{array}$} & \multicolumn{2}{|c|}{ Heterosis } \\
\hline & & & Gramos & $\%$ \\
\hline Perú - Andina & 66.54 & $65.27^{1}$ & -1.27 & -1.9 \\
\hline Perú - Inti & 66.62 & $65.28^{2}$ & -1.34 & -2.0 \\
\hline Andina - Inti & 66.83 & n.d. & n.d. & n.d. \\
\hline
\end{tabular}

n.d.= No se determinó

${ }^{1}$ Promedio fenotípico de los cruzamientos recíprocos (PxA y AxP)

${ }^{2}$ Promedio fenotípico del cruce que tuvo al genotipo Perú como vía paterna 
Cuadro 8. Promedios fenotípicos corregidos para el carácter grasa de deposición (\%) según el grupo genético

\begin{tabular}{lccc}
\hline $\begin{array}{l}\text { Genotipo no } \\
\text { hibrido (padres) }\end{array}$ & \multicolumn{3}{c}{ Genotipo no híbrido (madres) } \\
\cline { 2 - 4 } & Perú (P x P) & Andina (A x A) & Inti (I x I) \\
\hline Perú (P x P) & P x P & P x A & P x I \\
& $(0.53)$ & $(0.53)$ & $(0.48)$ \\
Andina (A x A) & $\mathrm{A} \times$ P & A x A & A x I \\
& $(0.48)$ & $(0.52)$ & (n.d) \\
Inti (I x I) & I x P & I x A & I x I \\
& (n.d) & (n.d) & $(0.57)$ \\
\hline
\end{tabular}

n.d.= No se determinó

Cuadro 9. Heterosis individual promedio estimado para el carácter grasa de deposición (\%)

\begin{tabular}{lcccc}
\hline \multirow{2}{*}{ Genotipos } & $\begin{array}{c}\text { Promedio de la } \\
\text { descendencia no } \\
\text { híbrida }\end{array}$ & \begin{tabular}{c} 
Promedio de la \\
descendencia \\
\cline { 5 - 6 }
\end{tabular} & 0.53 & \multicolumn{2}{c}{ Heterosida } \\
\hline Perú - Andina & 0.55 & $0.50^{1}$ & -0.03 & -5.7 \\
Perú - Inti & 0.55 & $0.48^{2}$ & -0.07 & -12.7 \\
Andina - Inti & & n.d. & n.d. & n.d. \\
\hline
\end{tabular}

n.d.= No se determinó

${ }^{1}$ Promedio fenotípico de los cruzamientos recíprocos (PxA y AxP)

${ }^{2}$ Promedio fenotípico del cruce que tuvo al genotipo Perú como vía paterna

Cuadro 10.Promedios fenotípicos corregidos para el carácter conversión alimenticia según el grupo genético

\begin{tabular}{lccc}
\hline $\begin{array}{l}\text { Genotipo no } \\
\text { hibrido (padres) }\end{array}$ & \multicolumn{3}{c}{ Genotipo no híbrido (madres) } \\
\cline { 2 - 4 } & Perú (P x P) & Andina (A x A) & Inti (I x I) \\
\hline Perú (P x P) & $\mathrm{P} \times \mathrm{P}$ & $\mathrm{P} \times \mathrm{A}$ & $\mathrm{P} \times \mathrm{I}$ \\
& $(3.26)$ & $(3.44)$ & $(3.46)$ \\
Andina (A x A) & $\mathrm{A} \times \mathrm{P}$ & $\mathrm{A} \times \mathrm{A}$ & $\mathrm{A} \times \mathrm{I}$ \\
& $(3.48)$ & $(3.18)$ & (n.d) \\
Inti (I x I) & $\mathrm{I} \times \mathrm{P}$ & $\mathrm{I} \times \mathrm{A}$ & $\mathrm{I} \times \mathrm{I}$ \\
& $(\mathrm{n} . \mathrm{d})$ & $($ n.d) & $(3.15)$ \\
\hline
\end{tabular}

n.d.= No se determinó 
Cuadro 11.Heterosis individual promedio estimado para el carácter velocidad de crecimiento

\begin{tabular}{lcccc}
\hline \multirow{2}{*}{ Genotipos } & $\begin{array}{c}\text { Promedio de la } \\
\text { descendencia no } \\
\text { híbrida }\end{array}$ & \begin{tabular}{c} 
Promedio de la \\
descendencia \\
\cline { 4 - 5 }
\end{tabular} & 3.22 & \multicolumn{2}{c}{ Heterosida } & Gramos & $\%$ \\
\hline Perú - Andina & 3.21 & $3.46^{1}$ & 0.24 & 7.4 \\
Perú - Inti & 3.17 & $3.46^{2}$ & 0.25 & 7.8 \\
Andina - Inti & & n.d. & n.d. & n.d. \\
\hline
\end{tabular}

n.d.= No se determinó

${ }^{1}$ Promedio fenotípico de los cruzamientos recíprocos (PxA y AxP)

${ }^{2}$ Promedio fenotípico del cruce que tuvo al genotipo Perú como vía paterna

Comparativamente, los niveles de heterosis individual encontrado para el carácter en referencia suelen ser similares a lo reportado por Muscari et al. (1994) en híbridos de ocho semanas, resultado de los de cruces Perú, Andina e Inti; sin embargo, suele ser inferior a lo reportado por Manosalvas et al. (2010) en híbridos resultante del cruce de otras líneas genéticas. Esta situación podría deberse a que las distancias genéticas de genotipos no híbridos considerados como parentales puros, no lo sean y tengan cierto grado de hibridación antepasada como para reducir las diferencias en las frecuencias alélicas de los genes asociados con la expresión del carácter en cuestión (Gardner et al., 1999; Angulo, 2013). A pesar de ello, el nivel de heterosis individual obtenido en los cruces efectuados resultan ser concordantes con lo reportado en otras especies de interés zootécnico, tal como refiere Magofke y García (2002), quienes refieren que la heterosis individual en características relacionadas con el crecimiento suelen ser bajas e inferiores al $7 \%$.

\section{Velocidad de Crecimiento}

Los promedios fenotípicos corregidos para el carácter velocidad de crecimiento a nivel de los grupos genéticos evaluados, así como los respectivos desvíos fenotípicos de los genotipos cruzados respecto al promedio de los parentales no híbridos se presentan en los cuadros 4 y 5. La descendencia PxI tuvo el mejor promedio fenotípico ( $10.3 \mathrm{~g} /$ día $)$ en relación al cruce $\operatorname{PxA}(10.0 \mathrm{~g} / \mathrm{día})$ y su reciproco $\operatorname{AxP}$ (9.2 g/día), lo cual implica un desvío fenotípico o heterosis individual de $0.395 \mathrm{~g} /$ día $(3.9 \%), 0.065 \mathrm{~g} /$ día $(0.7 \%)$ y $-0.675 \mathrm{~g} /$ día (-7.3\%), respectivamente. Por otro lado, la heterosis individual promedio obtenida en los cruzamientos recíprocos PxA y AxP fue de $-0.29 \mathrm{~g} /$ día (-3.0\%), resultando ser indeseable desde el punto de vista productivo y económico.

La heterosis individual promedia encontrada para este carácter a nivel de los tres grupos de descendientes híbridos F1 resultó ser ínfima a nivel del cruce PxI y negativo para el cruce PxA y su reciproco AxP, lo que indicaría la no conveniencia de realizar cruces basado en la utilización de ambos grupos genéticos si el objetivo es la optimización de la precocidad; sin embargo, el cruce que tuvo al genotipo Perú como vía paternal y al genotipo Inti como vía maternal ofrecería mayores posibilidades para conseguir dicho objetivo.

Los desvíos fenotípicos en sentido negativo registrado en el cruzamiento PxA y su recíproco $\mathrm{AxP}$ se deben probablemente al efecto de las interacciones interalélicas no complementarias o de tipo epistático involucrados en la expresión del carácter 
(Gardner et al., 1999; Magofke y García, 2002), no resultando ser ventajoso desde el punto de vista productivo.

\section{Rendimiento de Carcasa}

En los cuadros 6 y 7 se presentan los promedios fenotípicos corregidos para el carácter rendimiento de carcasa a nivel de los grupos genéticos, así como los respectivos desvíos fenotípicos de los genotipos cruzados respecto al promedio de los parentales no híbridos. Se puede observar que la descendencia de los cruzamientos tuvo promedios fenotípicos inferiores a la descendencia de los genotipos no híbridos, cuyos valores fueron de 65.4, 65.3 y $65.2 \%$ para los cruces PxA, PxI y AxP, respectivamente, los cuales representan desvíos fenotípicos desfavorables de $-1.16,-1.34$ y -1.38 , respecto al promedio fenotípico de los parentales no híbridos que le dieron origen.

Estos resultados estarían indicando la no manifestación de heterosis individual favorable a nivel de los cruces efectuados, y que los desvíos fenotípicos negativos serían promovidos por las interacciones génicas con efectos no complementarios de tipo epistático, repercutiendo desfavorablemente en la mejora genética de este carácter (Gardner et al., 1999, Falconer y Mackay, 2001). Otra posibilidad sería que los animales usados como línea pura en los cruzamientos efectuados tengan un alto grado de heterocigosidad como resultado de una incipiente selección o de algún tipo de hibridación, lo que reduciría las posibilidades de expresión de heterosis en la descendencia híbrida (Falconer y Mackay, 2001). Por tanto, el cruzamiento no sería el medio más adecuado como para propiciar la mejora de este carácter, quedando la selección como alternativa más apropiada para mejorarlo genéticamente.

Las escasas experiencias de trabajos en cruzamientos de cuyes en el país, y que además emplearon genotipos diferentes a los evaluados en el presente estudio, reportan medias fenotípicas cuyos desvíos respecto a los parentales no híbridos que le dieron origen suelen ser muy ínfimos e, incluso, resultaron estar por debajo de sus respectivas medias fenotípicas (Cruz, 2013; Gavilánez, 2014).

\section{Grasa de Deposición}

Los promedios fenotípicos corregidos para el carácter grasa de deposición, medido y extraído a nivel de la cruz y el cuello de los grupos genéticos evaluados, así como los respectivos desvíos de las medias fenotípicas de los genotipos cruzados respecto al promedio de los parentales no híbridos se presentan en los cuadros 8 y 9. La descendencia hibrida PxI y AxP tuvo un promedio fenotípico de 0.48 y $0.48 \%$, respectivamente, siendo ambos inferiores a la descendencia híbrida PxA cuya media fenotípica fue de $0.53 \%$. Este resultado implicaría un desvío fenotípico favorable en la deposición de grasa de $-0.07 \%$ para el cruce PxI y de $-0.03 \%$ para el cruce recíproco PxA y AxP, respecto a los parentales no híbridos que le dieron origen.

Los desvíos fenotípicos negativos obtenidos en la descendencia híbrida respecto a la descendencia de los parentales puros que le dieron origen indicarían, al igual que para el carácter rendimiento de carcasa, que los alelos de los loci involucrados en la expresión del carácter suelen tener interacciones génicas con efectos no complementarios, o de tipo epistático, o bien sus alelos tener una reducida frecuencia en la población, dando lugar a una reducción en la magnitud de la expresión del carácter en referencia a nivel de los híbridos (Gardner et al., 1999). Esta situación podría constituir un aspecto positivo y favorable para el consumidor, en tanto la tendencia sea a preferir carcasas con un menor contenido graso (Gil, 2007). Por tanto, mediante la hibridación, se estaría contribuyendo en la mejora de la calidad de la carcasa a razón de disminución de la proporción de ácidos grados saturados, aspecto que podría potenciarse con el manejo alimentario y dietas enriquecidas con insumos oleosos para 
mejora del perfil de ácidos grasos (Huamaní et al., 2016).

No se han reportado experiencias de trabajos de cruzamientos en cuyes en donde se haya evaluado el carácter en referencia; por tanto, esta situación resulta ser desventajoso para la pertinencia de los resultados del presente estudio, al no permitir contrastar o discrepar el aporte de la hibridación en la reducción del tejido graso subcutáneo. A pesar de ello, los resultados de la heterosis en sentido opuesto podrían resultar favorables para contribuir con la mejora de la presentación de las carcasas de los animales beneficiados, puesto que la tendencia actual es producir carnes con menor contenido de grasa acumulada (Gil, 2007).

\section{Conversión Alimenticia}

En los cuadros 10 y 11 se presentan los promedios fenotípicos corregidos para el carácter conversión alimenticia a nivel de los grupos genéticos evaluados, así como los respectivos desvíos fenotípicos de los genotipos cruzados respecto al promedio de los parentales no híbridos. Se puede observar que de los cruzamientos efectuados, la descendencia hibrida PxA, PxI y AxP tuvieron promedios fenotípicos de $3.44,3.46$ y 3.48 , respectivamente, lo que implicó desvíos fenotípicos desfavorables de $0.22,0.26$ y 0.26 respecto a la descendencia de los parentales no híbridos que le dieron origen, cuyos valores medios fueron 3.26, 3.18 y 3.15, tanto para los genotipos PxP, AxA y IxI, respectivamente. El desvío fenotípico registrado a nivel del cruzamiento reciproco PxAy AxP fue de 0.25 , respecto a la descendencia de los parentales no híbridos que le dieron origen.

Los promedios fenotípicos encontrados en el presente estudio y para el carácter conversión alimenticia, medida desde el destete hasta las 10 semanas de edad, contrastan con los reportes de Cruz (2013) y Gavilánez (2014) en cuyes de cruces de diversas líneas genéticas, quienes señalan que algunos cruces suelen tener mejores niveles de conver- sión alimenticia frente a otros tipos de cruces, quienes también resultaron ser inferiores a los parentales puros.

Por tanto, los resultados del presente estudio estarían evidenciando una menor eficiencia de los genotipos cruzados para convertir el alimento en carne en relación a los parentales no híbridos, de allí la inconveniencia de utilizar el cruzamiento como medio para mejorar genéticamente este carácter en particular, al igual que el rendimiento de carcasa, quedando la selección como recurso eficaz para propiciar la mejora genética de estos caracteres (Rivas, 2014).

Las diferencias y discrepancias encontrados entre los resultados del presente estudio y lo que reportan otras experiencias podrían explicarse por las diferencias en el manejo y control ambiental realizado durante la crianza, como es el caso del manejo de grupos contemporáneos como recurso para minimizar y controlar estos efectos ambientales (Gutiérrez, 2010). En otros estudios no se explicita el uso de metodologías tendientes a estimar factores de corrección para los efectos ambientales sistemáticos que resulten significativos con miras a efectuar el respectivo ajuste de datos. Asimismo, es posible que los genotipos usados en este estudio considerados como puros no lo sean, puesto que en la práctica no se pudo establecer con precisión la procedencia $u$ origen de los parentales comerciales Perú, Inti y Andina. Esto podría suponer que el grado de homocigosidad y las distancias genéticas de los animales considerados como puros no sean lo suficientemente altas como para permitir una mayor expresión del vigor hibrido (Avilés, 2016).

\section{Conclusiones}

- La heterosis individual estimada en los caracteres peso vivo y velocidad de crecimiento a partir de los genotipos comerciales Perú, Andina e Inti resulta ser ínfima y de poca relevancia productiva; $\sin$ 
embargo, existe una favorable contribución del efecto maternal del genotipo Andina e Inti sobre la mejora fenotípica de estos caracteres.

- El cruzamiento basado en el uso de las líneas comerciales Perú, Andina e Inti no produce efectos favorables sobre la mejora de la eficiencia a la conversión alimenticia y el rendimiento de carcasa, pero favorece la reducción del cúmulo de grasa depositada en ciertas partes del cuerpo del animal.

- La escasa manifestación de heterosis individual a nivel las características evaluadas sugieren que el método de cruzamiento sea usado en complementariedad de atributos de grupos genéticos diferenciados, dejando la selección como método de mayor eficacia para favorecer la mejora genética de los atributos de importancia económica en la producción de cuyes.

\section{Agradecimientos}

Los autores agradecen a la E.E. Canaán INIA-Ayacucho por el respaldo económico y los recursos brindados, así como al Instituto de Investigación de la Facultad de Ciencias Agrarias (IIFCA) por el estímulo ofrecido para concretar este estudio.

\section{Literatura Citada}

1. Angulo M. 2013. Aptitud combinatoria, efectos maternos y heterosis de los caracteres peso y tamaño de carnada en cruzamientos de líneas exóticas y poblaciones locales del Centro MEJOCUY. Tesis de Ing. Agrónomo. Cochabamba, Bolivia: Univ. Mayor de San Simón. 103 p.

2. Avilés ED. 2016. Caracterización genética del cuy doméstico en América del Sur mediante marcadores moleculares. Tesis Doctoral. Córdova, España: Univ. de Córdova. 125 p.
3. Bianchi A, Garibotto G, Bentancur O. 2001. Evaluación de la sobrevivencia, características de crecimiento, peso de la canal y punto GR en corderos pesados Corriedale puros y cruza Texel, Hampshire Down, Southdown y Suffolk. Arch Med Vet 33: 261-268. doi: 10.4067/ S0301-732X2001000200016

4. Cardellino R, Rovira J. 1987. Mejoramiento genético animal. Montevideo: Hemisferio Sur. $251 \mathrm{p}$.

5. Chauca FL. 2007. Logros obtenidos en la mejora genética del cuy (Cavia porcellus). Experiencias del INIA. En: XX Reunión ALPA. Cusco, Perú.

6. Chauca FL, Muscari J. Hirahona R. 2008. Investigación en cuyes. Tomo II. Lima: INIA. $155 \mathrm{p}$.

7. Cruz M. 2013. Comportamiento productivo de progenies F2 de cuatro cruzamientos entre grupos raciales de cuyes (Cavia porcellus) de hembras F1 con machos macabeo y peruano mejorado. Tumbaco-Pichincha. Tesis de Ing. Agrónomo. Quito: Univ. Central del Ecuador. $63 \mathrm{p}$.

8. Dulanto M. 1999. Parámetros productivos y reproductivos de tres líneas puras y dos grados de cruzamiento entre líneas de cuyes. Tesis de Bachiller. Lima: Univ. Nacional Agraria La Molina. 65 p.

9. Falconer SD, Mackay CT. 2001. Introducción a la genética cuantitativa. Zaragoza, España: Acribia. 490 p.

10. Gardner E, Simmons M, Snustad P. 1999. Principios de genética. $4^{\text {a }}$ ed. México: Ed Limusa. 649 p.

11. Gavilánez F. 2014. Análisis productivo de las progenies F2 y F3 de cuatro cruzamientos entre grupos raciales de cuyes (Cavia porcellus), macabeo y peruano mejorado. Tumbaco, Pichincha. Tesis de Ing. Agrónomo. Quito: Univ. Central del Ecuador. $75 \mathrm{p}$.

12. Gil V. 2007. Importancia del cuy y su competitividad en el mercado. Arch Latinoam Prod Anim 15: 216. 
13. Gutiérrez JP. 2010. Iniciación a la valoración genética animal. Metodología adaptada al EEES. España: UCM Ed Complutense. 355 p.

14. Huamaní G, Zea O, Gutiérrez R, Vilchez C. 2016. Efecto de tres sistemas de alimentación sobre el comportamiento productivo y perfil de ácidos grasos de carcasa de cuyes (Cavia porcellus). Rev Inv Vet Perú 27: 486494. doi: 10.15381/rivep.v27i3.12004

15. Magofke J, García C. 2001. Uso del cruzamiento entre razas para mejorar la productividad en animales. IV. Diseño y comparaciones requeridas para estimar el mérito de las razas puras usadas en cruzamiento y el vigor hibrido directo y materno. Circular de extensión. Santiago de Chile: Univ. de Chile. 12 p.

16. Magofke J, García C. 2002. Uso del cruzamiento entre razas para mejorar la productividad animal I: Conceptos. Circular de extensión. Santiago de Chile: Univ. de Chile. 8 p.

17. Manosalvas C, León V, Lalama $\boldsymbol{H}$. 2010. Evaluación de la progenie (F1) de cuatro cruzamientos de dos grupos raciales de cuyes (Cavia porcellus) entre
Peruano Mejorado con Macabeo. Tumbaco-Pichincha. Tesis de Ing. Agrónomo. Quito: Univ. Central del Ecuador. $95 \mathrm{p}$.

18. Matlab. 2007. The language of technical computing. MathWorks. [Internet]. Available in: https://www.mathworks.com/tagteam/73554_91199v02_overview.pdf

19. Muscari J. 1993. Avance de investigación en el mejoramiento genético del cuy. En: IV Congreso Latinoamericano de Cuyicultura. Riobamba, Ecuador.

20. Muscari J, Higaonna R, Chauca L. 1994. Heterosis obtenida mediante cruzamiento de tres líneas de cuyes (Cavia porcellus). En XVII En: Reunión Científica de la Asociación Peruana de Producción Animal. Lima, Perú.

21. Rivas C. 2014. Distinción de rasgos genéticos en líneas de cuyes. Info INIAF 1(3): 38-46.

22. Rivas C, Rico E. 2014. Plan de conservación, manejo sostenible y mejora genética de cuyes en Bolivia. Proyecto ASDI - DICyT 15/27 UMSS- Centro MEJOCUY: 2009. p 19- 21. 